This article was downloaded by: [Institute of Mechanics]

On: 18 November 2013, At: 00:00

Publisher: Taylor \& Francis

Informa Ltd Registered in England and Wales Registered Number: 1072954

Registered office: Mortimer House, 37-41 Mortimer Street, London W1T 3J H, UK

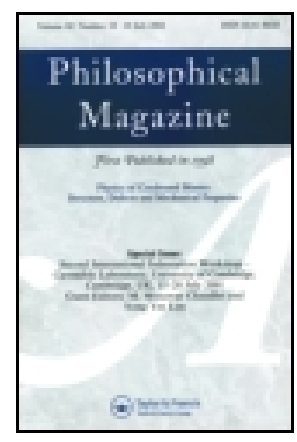

Philosophical Magazine A

Publication details, including instructions for authors and subscription information:

http:// www.tandfonline.com/loi/ tpha20

\title{
Dislocation theory of the fracture criterion for anisotropic solids
}

\author{
T. C. Wang ${ }^{\text {a }}$ \\ a LNM, Institute of Mechanics, Chinese Academy of \\ Sciences, 100080, Beijing, PR China \\ Published online: 12 Aug 2009.
}

To cite this article: T. C. Wang (1998) Dislocation theory of the fracture criterion for anisotropic solids, Philosophical Magazine A, 77:1, 31-53, DOI: 10.1080/01418619808214229

To link to this article: http:// dx.doi.org/ 10.1080/01418619808214229

\section{PLEASE SCROLL DOWN FOR ARTICLE}

Taylor \& Francis makes every effort to ensure the accuracy of all the information (the "Content") contained in the publications on our platform. However, Taylor $\&$ Francis, our agents, and our licensors make no representations or warranties whatsoever as to the accuracy, completeness, or suitability for any purpose of the Content. Any opinions and views expressed in this publication are the opinions and views of the authors, and are not the views of or endorsed by Taylor \& Francis. The accuracy of the Content should not be relied upon and should be independently verified with primary sources of information. Taylor and Francis shall not be liable for any losses, actions, claims, proceedings, demands, costs, expenses, damages, and other liabilities whatsoever or howsoever caused arising directly or indirectly in connection with, in relation to or arising out of the use of the Content.

This article may be used for research, teaching, and private study purposes. Any substantial or systematic reproduction, redistribution, reselling, loan, sub-licensing, systematic supply, or distribution in any form to anyone is expressly forbidden.

Terms \& Conditions of access and use can be found at http://www.tandfonline.com/ page/terms-and-conditions 
Philosophical Magazine A, 1998, Vol. 77, No. 1, 31-53

\title{
Dislocation theory of the fracture criterion for anisotropic solids
}

\author{
By T. C. WANG \\ LNM, Institute of Mechanics, Chinese Academy of Sciences, 100080, Beijing, \\ PR China
}

[Received 2 July 1996 and accepted in revised form 21 April 1997]

\begin{abstract}
Abstr aCt
A dislocation theory of fracture criterion for the mixed dislocation emission and cleavage process in an anisotropic solid is developed in this paper. The complicated cases involving mixed-mode loading are considered here. The explicit formula for dislocations interaction with a semi-infinite crack is obtained. The governing equation for the critical condition of crack cleavage in an anisotropic solid after a number dislocation emissions is established. The effects of elastic anisotropy, crack geometry and load phase angle on the critical energy release rate and the total number of the emitted dislocations at the onset of cleavage are analysed in detail. The analyses revealed that the critical energy release rates can increase to one or two magnitudes larger than the surface energy because of the dislocation emission. It is also found elastic anisotropy and crystal orientation have significant effects on the critical energy release rates. The anisotropic values can be several times the isotropic value in one crack orientation. The values may be as much as $40 \%$ less than the isotropic value in another crack orientation and another anisotropy parameter. Then the theory is applied to a fcc single crystal. An edge dislocation can emit from the crack tip along the most highly shear stressed slip plane. Crack cleavage can occur along the most highly stressed slip plane after a number of dislocation emissions. Calculation is carried out step by step. Each step we should judge by which slip system is the most highly shear stressed slip system and which slip system has the largest energy release rate. The calculation clearly shows that the crack orientation and the load phase angle have significant effects on the crystal brittle-ductile behaviours.
\end{abstract}

\section{$\S 1$. INTRODUCTION}

A dislocation theory of crack propagation in an anisotropic solid due to dislocation emission and cleavage is proposed in this paper. The concepts adopted here have been developed by Rice and Thomson (1974), Ohr (1985) and Lin and Thomson (1986). The well known dislocation emission model proposed by Rice and Thomson (1974) gave a quantitative criterion for ductile as against brittle behaviour. Beltz and Rice (1991), Schoeck (1991), Rice (1992), Rice et al. (1992), Wang (1995) have reanalysed the Rice-Thomson model on the basis of the Peierls framework, in which the fully emitted dislocation is considered as a continuous distribution of infinitesimal continuum dislocations. For the mode II case, Rice (1992) presented an exact solution for the critical loading at that nucleation instability was developed and identified a solid-state parameter, the unstable stacking energy $\gamma_{\text {us }}$, which characterizes the resistance to dislocation nucleation.

The analyses have also been generalized to anisotropic solids by Sun and Beltz (1994). They found the anisotropic critical crack extension force for dislocation emission may be greater or less than its isotropic counterpart. For bec $\alpha$-Fe, the anisotropic 
values can be 2.4 times larger than the isotropic values in one crack orientation; in another crack orientation, the values are as much as $40 \%$ less than the isotropic values.

The brittle cleavage of a crack in metal is usually accompanied by a considerable number of dislocation emissions. A fracture criterion accounting for the effects of the dislocation emission was proposed by Sinclair and Finnis (1983) with a simple analysis for a pure mode I crack. Their model was constrained to cleavage on one plane and crack branching was ruled out. A general theory for crack propagation in the situation of combined cleavage and dislocation emission was developed by Lin and Thomson (1986). Their model is also constrained to cleavage on one plane and crack branching was not permitted.

From numerous observations, Ohr (1985) pointed out that crack propagation was a mixed-mode process in which the dislocation emission and the cleavage could proceed in the same plane.

A general theory for crack propagation due to combined dislocation emission and cleavage for isotropic solid was proposed by Wang (1996) based on Ohr's model. This analysis is generalized to take into account elastic anisotropy in this paper. The explicit formula is obtained for the critical condition of crack cleavage propagation in an anisotropic solid after a number of dislocation emissions. The effects of the elastic anisotropy, the crack geometry and the load phase angle on the critical energy release rates and the total number of the emitted dislocations at the onset of cleavage are analysed in detail. The analyses revealed that the critical energy release rates can increase to one or two magnitudes larger than the surface energy owing to the dislocation emission. it is also found that elastic anisotropy and crystal orientation have a significant effect on the critical energy release rates. The anisotropic values can be several times the isotropic value in one crack orientation. The values may be as much as $40 \%$ less than the isotropic value in another crack orientation and another anisotropy parameter. Then the general theory is applied to the fcc single crystal. 12 possible slip systems reduce to three effective slip systems for the plane-strain problem. An edge dislocation can be emitted from the crack tip along the most highly shear stressed slip plane. Crack cleavage can occur along the most highly stressed slip plane, in which the energy release rate reaches the maximum value, after a number of dislocation emissions. The calculation clearly shows that the crack orientation and the load phase angle have significant effects on the crystal brittle-ductile behaviours.

\section{§2. REPRESENTATION AND FORMULAE}

A complex-variable formulation that represents the stress and displacements in an anisotropic solid was developed by Esheby et al. (1953), Stroh (1958) and Lekhnitskii (1963). A brief review of this presentation was given by Suo (1989).

The representation for displacements and stresses is

$$
\begin{aligned}
\sigma_{2 i} & =2 \operatorname{Re}\left(\sum_{j=1}^{3} L_{i j} \Phi_{j}\left(z_{j}\right)\right), \\
\sigma_{1 i} & =-2 \operatorname{Re}\left(\sum_{j=1}^{3} L_{i j} \mu_{j} \Phi_{j}\left(z_{j}\right)\right), \\
u_{i} & =2 \operatorname{Re}\left(\sum_{j=1}^{3} A_{i j} \phi_{j}\left(z_{j}\right)\right), \quad \Phi(z)=\phi^{\prime}(z),
\end{aligned}
$$


Fig. 1

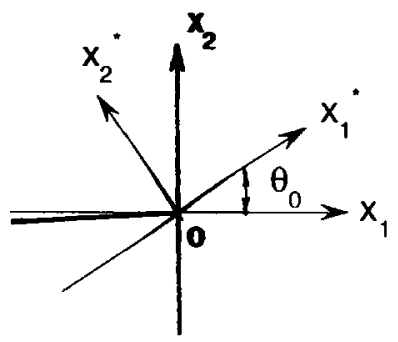

Geometry of the crack plane and the most shear stressed slip plane.

where $\mathbf{A}$ and $\mathbf{L}$ are the matrices which are determined by elastic constants, $z_{j}=$ $x_{1}+\mu_{j} x_{2}, \mu_{j}(j=1,2,3)$ are the characteristic numbers. We only consider plane problem. Two-dimensional theory is proposed here. The coordinate system is as follows: the $x_{1}$ axis is the crack elongation direction, $x_{2}$ the normal to the crack plane and $x_{3}$ along the crack front. Assume that the $x_{1}-x_{2}$ plane is a mirror plane for the anisotropic solid, so that the in-plane field quantities are decoupled from antiplane quantities.

Suppose that the crack front is contained within one slip plane which is most highly stressed in an anisotropic crystal. As shown in fig. 1, the slip plane makes an inclined angle with respect to the crack plane.

For a crack propagating in the $x_{1}$ direction in an anisotropic solid, the energy release rate has been given by Stroh (1958) and Sih et al. (1965):

$$
G=\frac{k^{\mathrm{T}}(B+\bar{B}) k}{4}
$$

where $\mathbf{k}=\left[k_{1}, k_{2}, k_{3}\right]^{\mathrm{T}}=\left[k_{\mathrm{II}}, k_{\mathrm{I}}, k_{\mathrm{III}}\right]^{\mathrm{T}}$ is the stress intensity factor, $\mathbf{B}$ is the Hermitian matrix:

$$
\mathbf{B}=\mathbf{i A L} \mathbf{L}^{-1}
$$

The overbar denotes the complex conjugation. The singular stress fields can be expressed by the complex potential (Sih et al. 1965, Suo 1989, 1990)

$$
\Phi_{i}(z)=\sum_{j=1}^{3} L_{i j}^{-1} \frac{k_{j}}{2(2 \pi z)^{1 / 2}} .
$$

Substituting eqn. (4) into eqn. (1), one obtains

$$
\sigma_{2 i}=\operatorname{Re}\left(\sum_{j=1}^{3} \sum_{n=1}^{3} L_{i j} L_{j n}^{-1} k_{n} /\left(2 \pi z_{j}\right)^{1 / 2}\right) .
$$

Along the $x_{1}$ direction, we have $z_{j}=x_{1}(j=1,2,3)$; hence we get

$$
\sigma_{2 i}=\frac{k_{i}}{\left(2 \pi x_{1}\right)^{1 / 2}} \text {. }
$$


Introducing a new coordinate system o $x_{1}^{*} x_{2}^{*} x_{3}^{*}$ : the $x_{1}^{*}$ axis is the slip plane, $x_{2}^{*}$ normal to the slip plane and $x_{3}^{*}$ coincident with $x_{3}$. Now we have an in-plane coordinate rotation

$$
\mathbf{R}=\left[\begin{array}{ccc}
\cos \theta_{0} & \sin \theta_{0} & 0 \\
-\sin \theta_{0} & \cos \theta_{0} & 0 \\
0 & 0 & 1
\end{array}\right]
$$

where $\theta_{0}$ is the angle measured from the $x_{1}$ axis to the $x_{1}^{*}$ axis. Under such an inplane rotation, $\mathbf{B}$ transforms as a second-order tensor and each column of $\mathbf{A}$ and $\mathbf{L}$ transforms as a vector, that is

$$
\begin{aligned}
& \mathbf{A}^{*}=\mathbf{R A}, \\
& \mathbf{L}^{*}=\mathbf{R} \mathbf{L}, \\
& \mathbf{B}^{*}=\mathbf{R B} \mathbf{R}^{\mathrm{T}} .
\end{aligned}
$$

The traction $\mathbf{p}$, which acts on the slip plane, can be represented as

$$
\begin{aligned}
p_{i}=\sigma_{i j} n_{j}=-\sigma_{i 1} \sin \theta_{0}+\sigma_{i 2} \cos \theta_{0} & =2 \operatorname{Re}\left(\sum_{j=1}^{3} L_{i j}\left(\mu_{j} \sin \theta_{0}+\cos \theta_{0}\right) \Phi_{j}\left(z_{j}\right)\right) \\
& =2 \operatorname{Re}\left(\sum_{j=1}^{3} L_{i j} \omega_{j} \Phi_{j}\left(z_{j}\right)\right) .
\end{aligned}
$$

Substituting eqn. (4) into eqn. (9), one obtains

$$
p_{i}=\frac{1}{(2 \pi r)^{1 / 2}} \operatorname{Re}\left(\sum_{m=1}^{3} \sum_{n=1}^{3} L_{i m} \omega_{m}^{1 / 2} L_{m n}^{-1} k_{n}\right)
$$

where

$$
\omega_{j}=\cos \theta_{0}+\mu_{j} \sin \theta_{0}
$$

Transforming to the new coordinate system $o x_{1}^{*} x_{2}^{*} x_{3}^{*}$, the traction component $p_{i}^{*}$ becomes

$$
p_{i}^{*}=\frac{1}{(2 \pi r)^{1 / 2}} \operatorname{Re}\left(\sum_{j=1}^{3} \sum_{m=1}^{3} \sum_{l-1}^{3} R_{i j} L_{j m} \omega_{m}^{1 / 2} L_{m l}^{-1} k_{l}\right) .
$$

With respect to the coordinate system $o x_{1}^{*} x_{2}^{*} x_{3}^{*}$ one can define the stress intensity factor $\mathbf{K}=\left[K_{1}, K_{2}, K_{3}\right]^{\mathbf{T}}=\left[K_{\mathrm{II}}, K_{\mathrm{I}}, K_{\mathrm{III}}\right]^{\mathrm{T}}$ as follows:

$$
K_{i}=\lim _{r \rightarrow 0}\left[(2 \pi r)^{1 / 2} p_{i}^{*}\right]
$$

Hence we obtain

$$
K_{i}=\operatorname{Re}\left(\sum_{j=1}^{3} \sum_{m=1}^{3} \sum_{l=1}^{3} R_{i j} L_{j m} \omega_{m}^{1 / 2} L_{m l}^{-1} k_{l}\right)=\sum_{j=1}^{3} f_{i j} k_{j}
$$

where the coefficients $f_{i j}$ are

$$
f_{i j}=\operatorname{Re}\left(\sum_{k=1}^{3} \sum_{m=1}^{3} R_{i k} L_{k m} \omega_{m}^{1 / 2} L_{m j}^{-1}\right)
$$


The problem of an edge dislocation interacting with a crack in anisotropic solid was solved by Atkinson (1996), Asaro (1975) and Suo (1989). The formulae adopted here were given by Suo (1989), Wang et al. (1992) and Wang (1994).

The complex potential for an edge dislocation at point $\left(x_{0}, y_{0}\right)$ interacting with an semi-infinite crack can be expressed as

$$
\Phi_{i}^{\mathrm{d}}(z)=\frac{d_{i}}{z-s_{i}}-\frac{1}{2 z^{1 / 2}}\left(\frac{d_{i}}{z^{1 / 2}+s_{i}^{1 / 2}}+\sum_{j=1}^{3} C_{i j} \frac{\overline{d_{j}}}{z^{1 / 2}+\overline{s_{j}^{1 / 2}}}\right)
$$

where $s_{i}=x_{0}+\mu_{i} y_{0}$, vector $\mathbf{d}$ and matrix $\mathbf{C}$ are

$$
\begin{aligned}
& \mathbf{d}=\frac{1}{2 \pi} \mathbf{L}^{-1}(\mathbf{B}+\overline{\mathbf{B}})^{-1} \mathbf{b} \\
& \mathbf{C}=\mathbf{L}^{-1} \overline{\mathbf{L}}
\end{aligned}
$$

where $\mathbf{b}$ is the Burgers vector of the edge dislocation.

Equation (13) can be rewritten as follows:

$$
\Phi_{i}^{\mathrm{d}}(z)=\frac{s_{i}^{1 / 2}}{z^{1 / 2}} \frac{d_{i}}{z-s_{i}}+\frac{1}{2 z^{1 / 2}}\left(\frac{d_{i}}{z^{1 / 2}+s_{i}^{1 / 2}}-\sum_{j=1}^{3} C_{i j} \frac{\overline{d_{j}}}{z^{1 / 2}+\overline{s_{j}^{1 / 2}}}\right) .
$$

The traction $\mathbf{p}^{\mathrm{d}}$, produced by the edge dislocation and acting in the slip plane, is

$$
\begin{aligned}
p_{i}^{\mathrm{d}}= & \frac{r_{0}^{1 / 2}}{r^{1 / 2}} \frac{2}{r-r_{0}} \operatorname{Re}\left(\sum_{j=1}^{3} L_{i j} d_{j}\right)+\frac{1}{r^{1 / 2}}\left\{\left[\operatorname{Re}\left(\sum_{j=1}^{3} L_{i j} d_{j}\right)\right] /\left(r^{1 / 2}+r_{0}^{1 / 2}\right)\right. \\
& \left.-\operatorname{Re}\left(\sum_{k=1}^{3} \sum_{j=1}^{3} \frac{L_{i j} C_{j k} \overline{d_{k}}}{r^{1 / 2}+r_{0}^{1 / 2}\left(\bar{\omega}_{k} / \omega_{j}\right)^{1 / 2}}\right)\right\}
\end{aligned}
$$

where $r_{0}$ is the distance from the crack tip to the edge dislocation.

Transforming to the coordinate system $0 x_{1}^{*} x_{2}^{*} x_{3}^{*}$, one obtains

$$
p_{i}^{\mathrm{d} *}=\sum_{j=1}^{3} R_{i j} p_{j}^{\mathrm{d}}
$$

The stress intensity factors contributed by the edge dislocation in the coordinate system $o x_{1}^{*} x_{2}^{*} x_{3}^{*}$ can be defined as

$$
\begin{aligned}
K_{i}^{\mathrm{d}}=\lim _{r \rightarrow 0}\left[(2 \pi r)^{1 / 2} p_{i}^{\mathrm{d} *}\right]= & -\frac{(2 \pi)^{1 / 2}}{r_{0}^{1 / 2}} \operatorname{Re}\left(\sum_{j=1}^{3} \sum_{m=1}^{3} R_{i j} L_{j m} d_{m}\right. \\
& \left.+\sum_{j=1}^{3} \sum_{k=1}^{3} \sum_{m=1}^{3} R_{i j} L_{j m} C_{m k} \bar{d}_{k} \frac{\omega_{m}^{1 / 2}}{\bar{\omega}_{k}^{1 / 2}}\right) .
\end{aligned}
$$

Equation (17) can be represented as follows:

$$
K_{i}^{\mathrm{d}}=-\frac{q_{i}}{\left(2 \pi r_{0}\right)^{1 / 2}}
$$


where

$$
q_{i}=2 \pi \operatorname{Re}\left(\sum_{j=1}^{3} \sum_{m=1}^{3} R_{i j} L_{j m} d_{m}+\sum_{j=1}^{3} \sum_{k=1}^{3} \sum_{m=1}^{3} R_{i j} L_{j m} C_{m k} \bar{d}_{k} \frac{\omega_{m}^{1 / 2}}{\bar{\omega}_{k}^{1 / 2}}\right)
$$

The local stress intensity factors are given by

$$
\begin{aligned}
& K_{\mathrm{I}}^{\mathrm{tip}}=K_{\mathrm{I}}+K_{\mathrm{I}}^{\mathrm{d}}, \\
& K_{\mathrm{II}}^{\mathrm{tip}}=K_{\mathrm{II}}+K_{\mathrm{II}}^{\mathrm{d}}, \\
& K_{\mathrm{III}}^{\mathrm{tip}}=K_{\mathrm{III}}+K_{\mathrm{III}}^{\mathrm{d}} .
\end{aligned}
$$

The physical meaning of eqn. (20) is clear. The local stress intensity factors are less than the applied stress intensity factors. This decrease in local stress intensity factor is caused by the dislocation shielding.

When an edge dislocation is emitted from the crack tip along the slip plane, the emission condition has been proposed by Lin and Thomson (1986) and Rice (1992) as follows:

$$
K_{\mathrm{II}}^{\mathrm{tip}}=K_{\text {IIe }}
$$

where $K_{\text {IIe }}$ is the critical stress intensity factor for the dislocation emission along the slip plane. According to Rice (1992),

$$
K_{\mathrm{Ile}}=\left(\frac{2 \mu_{\mathrm{eff}} \gamma_{\mathrm{us}}}{1-\nu_{\mathrm{eff}}}\right)^{1 / 2}
$$

where $\gamma_{\mathrm{us}}$ is the unstable stacking energy of the slip plane, $\mu_{\mathrm{eff}}$ is the effective shear modulus and $\nu_{\text {eff }}$ is the effective Poisson ratio.

The cleavage criterion for the crack branching into slip plane is given by

$$
G^{* \text { tip }}=\frac{\mathbf{K}^{\mathrm{tip} \mathrm{T}}(\mathbf{B}+\overline{\mathbf{B}})^{*} \mathbf{K}^{\mathrm{tip}}}{4}=G_{\mathrm{Ic}}
$$

where $G_{\mathrm{Ic}}$ is the fracture toughness for a crack propagating along the slip plan. We discuss only the in-plane problem here. Equation (23) can be expanded as

$$
a_{11}\left(K_{1}^{\mathrm{tip}}\right)^{2}+2 a_{12} K_{2}^{\mathrm{tip}}+a_{22}\left(K_{2}^{\mathrm{tip}}\right)^{2}=G_{\mathrm{Ic}}
$$

where $a_{i j}=(B+\bar{B})_{i j}^{*} / 4$. Meanwhile at the onset of cleavage branching, the local mode II stress intensity factor $K_{\mathrm{II}}^{\mathrm{tip}}$ is given by

$$
K_{\mathrm{II}}^{\mathrm{tip}}=\eta K_{\mathrm{II}}, \quad 0 \leqslant \eta \leqslant 1
$$

Suppose that the cleavage criterion (23) is met after $N$ edge dislocations were emitted. Then the local stress intensity factors are given by

$$
K_{j}^{\mathrm{tip}}=K_{j}-q_{j} \sum_{i=1}^{N} \frac{1}{\left(2 \pi r_{i}\right)^{1 / 2}}
$$


where $r_{i}(i=1,2, \ldots, N)$ is the distance from the crack tip of the $i$ th emitted dislocation. Introduce several parameters as follows:

$$
\begin{aligned}
\frac{1}{\left(2 \pi r_{\mathrm{c}}\right)^{1 / 2}} & =\frac{1}{N} \sum_{i=1}^{N} \frac{1}{\left(2 \pi r_{i}\right)^{1 / 2}}, \\
c_{j} & =\frac{q_{j}}{\left(2 \pi r_{\mathrm{c}}\right)^{1 / 2} K_{\mathrm{IIe}}}=q_{0} \frac{\left(s_{11} s_{22}\right)^{1 / 2} q_{j}}{b}, \\
q_{0} & =\frac{b}{\left(2 \pi r_{\mathrm{c}}\right)^{1 / 2} K_{\mathrm{IIe}}\left(s_{11} s_{22}\right)^{1 / 2}} .
\end{aligned}
$$

Equation (26) now becomes

$$
K_{i}^{\mathrm{tip}}=K_{i}-N c_{i} K_{\mathrm{Ile}}
$$

Substituting eqn. (28) into eqn. (24), one obtains

$a_{11}\left(K_{1}-N K_{\text {IIe }} c_{1}\right)^{2}+2 a_{12}\left(K_{1}-N K_{\text {IIe }} c_{1}\right)\left(K_{2}-N K_{\text {Ile }} c_{2}\right)+a_{22}\left(K_{2}-N K_{\text {IIe }} c_{2}\right)^{2}=G_{\text {Ic }}$

Equation (29) can be expanded as follows:

$$
\begin{aligned}
& N^{2}\left(a_{11} c_{1}^{2}+2 a_{12} c_{1} c_{2}+a_{22} c_{2}^{2}\right) K_{\mathrm{Ile}}^{2}-2 N\left[a_{11} c_{1} K_{1}+a_{12}\left(c_{1} K_{2}+c_{2} K_{1}\right)+a_{22} c_{2} K_{2}\right] K_{\mathrm{IIe}} \\
& \quad+\left(a_{11} K_{1}^{2}+2 a_{12} K_{1} K_{2}+a_{22} K_{2}^{2}\right)=G_{\mathrm{Ic}}=a_{22} K_{\mathrm{Ic}}^{2} .
\end{aligned}
$$

Equation (30) can be represented as follows:

where

$$
N^{2} c_{0}-2 N b_{0} \frac{K_{2}}{K_{\text {IIe }}}+a_{0}\left(\frac{K_{2}}{K_{\text {IIc }}}\right)^{2}=a_{22} \rho_{K}^{2}
$$

$$
\begin{aligned}
a_{0} & =a_{11} \tan ^{2} \psi+2 a_{12} \tan \psi+a_{22}, \\
b_{0} & =a_{11} c_{1} \tan \psi+a_{12}\left(c_{1}+c_{2} \tan \psi\right)+a_{22} c_{2}, \\
c_{0} & =a_{11} c_{1}^{2}+2 a_{12} c_{1} c_{2}+a_{22} c_{2}^{2}, \\
\tan \psi & =\frac{K_{1}}{K_{2}}=\frac{K_{\mathrm{II}}}{K_{\mathrm{I}}} \\
\rho_{K} & =\frac{K_{\mathrm{Ic}}}{K_{\mathrm{Ile}}} .
\end{aligned}
$$

$\psi$ defines the stress intensity ratio and $\rho_{K}$ characterizes the ratio of the fracture toughness to the critical stress intensity factor for dislocation emission from the crack tip along the slip plane. If we know the total number $N$ of the emitted dislocations, one can easily obtained the critical value $K_{2} / K_{\text {Ile }}$ from eqn. (31).

As shown in fig. 2, when the applied load is increased, the stress intensity factors $K_{1}$ and $K_{2}$ will simultaneously increase along the straight line OC. At point $\mathrm{A}_{0}$, the mode II stress intensity factor reaches the critical value $K_{\text {lle. The }}$ first dislocation is fully nucleated at the crack tip, then emitted from the crack tip along the slip plane and finally stopped at distance $r_{1}$. The local stress intensity factor $K_{1}^{\text {tip }}$ and $K_{2}^{\text {tip }}$ are decreased from point $\mathrm{A}_{0}$ to point $\mathrm{B}_{1}$ along the straight line $\mathrm{A}_{0} \mathrm{~B}_{1}$. The slope of the straight line $A_{0} B_{1}$ is determined by eqn. (17). As the applied load 
Fig. 2

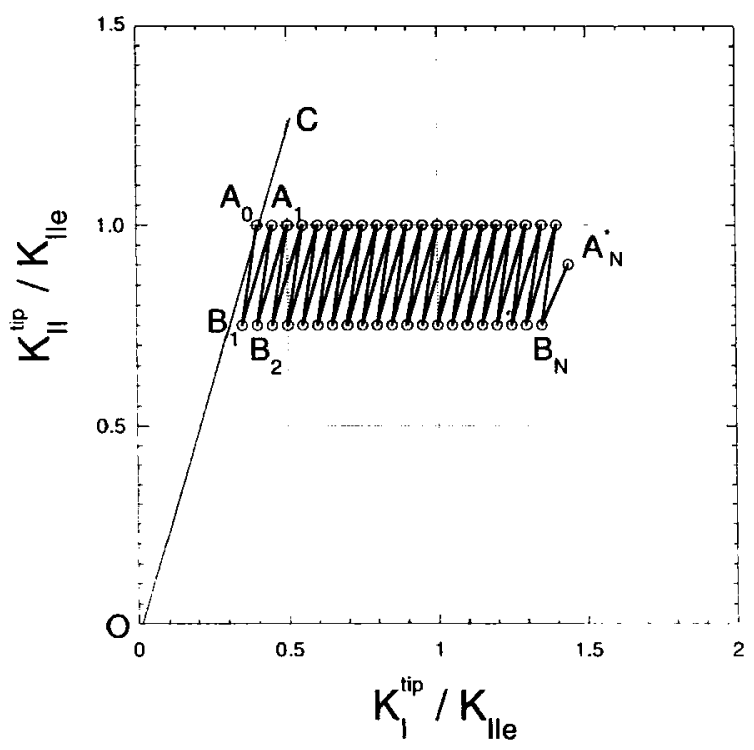

Effects of applied load and dislocation shielding on the local stress intensity factors.

increases again, the local stress intensity factors $K_{1}^{\text {tip }}$ and $K_{2}^{\text {tip }}$ increase along a straight line $B_{1} A_{1}$, which is parallel to the straight line $O C$. At point $A_{1}$, the local mode II stress intensity factor reaches the critical value $K_{\text {Ile }}$ again, the second dislocation is fully nucleated at the crack tip, then emitted along the slip plane and finally stopped at distance $r_{2}$. The local stress intensity factors decrease from point $A_{1}$ to $B_{2}$ owing to the second dislocation shielding. As the sequence was repeated, at the critical point $\mathrm{A}_{N}^{*}$, the radius $\mathrm{OA}_{N}^{*}$ is equal the radius $\mathrm{OC}$ after the $N$ th dislocation was emitted. The fracture criterion (31) is met and cleavage branching occurs.

\section{§3. RESULTS FOR ORTHOTROPIC SOLIDS}

In this section we survey only the anisotropic effects on the critical energy release rates for crack cleavage extension after a number dislocation emissions. The crystallographic structure is not introduced in this section. The calculation was carried out for orthotropic solids.

For a given orthotropic solid, only four elastic constants $s_{11}, s_{22}, s_{12}$ and $s_{66}$ enter the plane problem formulae. Introduce two non-dimensional parameters (Suo 1989)

$$
\lambda=\frac{s_{11}}{s_{22}} . \quad \rho=\frac{2 s_{12}+s_{66}}{2\left(s_{11} s_{22}\right)^{1 / 2}} .
$$

In an isotropic solid, Young's modulus along any direction would be the same but, for an anisotropic solid, different directions have different Young's moduli. The parameter $\lambda$ measures the anisotropy in the elastic Young's moduli in the $x_{1}$ direction and $x_{2}$ direction. The parameter $\rho$ measures the anisotropy in the shear modulus 
for the $x_{1}-x_{2}$ plane. For isotropic solids, $\lambda=\rho=1$. The characteristic equation is

$$
\lambda \mu^{4}+2 \rho \lambda^{1 / 2} \mu^{2}+1=0
$$

Hence the characteristic numbers depend on $\lambda$ and $\rho$ only. The characteristic numbers are

$$
\begin{aligned}
& \mu_{1}=\mathrm{i} \lambda^{-1 / 4}(n+m), \quad \mu_{2}=\mathrm{i} \lambda^{-1 / 4}(n-m), \quad 1 \leqslant \rho \leqslant \infty, \\
& \mu_{1}=\lambda^{-1 / 4}(\text { in }+m), \quad \mu_{2}=\lambda^{-1 / 4}(\text { in }-m), \quad-1 \leqslant \rho \leqslant 1 .
\end{aligned}
$$

The matrices $\mathbf{L}$ and $\mathbf{B}$ for an orthotropic solid with the $x-y$ plane as a mirror plane, are

$$
\begin{aligned}
& \mathbf{L}=\left[\begin{array}{ccc}
-\mu_{1} & -\mu_{2} & 0 \\
1 & 1 & 0 \\
0 & 0 & -1
\end{array}\right], \\
& \mathbf{B}=\left[\begin{array}{ccc}
2 n \lambda^{1 / 4}\left(s_{11} s_{22}\right)^{1 / 2} & \mathrm{i}\left[\left(s_{11} s_{22}\right)^{1 / 2}+s_{12}\right] & 0 \\
-\mathrm{i}\left[\left(s_{11} s_{2}\right)^{1 / 2}+s_{12}\right] & 2 n \lambda^{-1 / 4}\left(s_{11} s_{22}\right)^{1 / 2} & 0 \\
0 & 0 & \left(s_{44} s_{55}\right)^{1 / 2}
\end{array}\right] .
\end{aligned}
$$

The stress intensity factors $k_{1}$ and $k_{2}$ are taken as external loads. The load phase angle $\psi_{0}$ is defined as $\tan \psi_{0}=k_{1} / k_{2}$.

The calculation is carried out for the plane strain. The parameter $q_{0}$ is chosen as $q_{0}=0.054$.

Figure 3 shows the critical energy release rates for crack cleavage against load phase angle $\psi_{0}$ for different values of $\lambda$ when $\theta_{0}=0, \rho_{K}=2.0$ and $\rho=0.99999$. It is clear that the critical energy release rate is markedly increased as the load phase angle $\psi_{0}$ increases. On the other hand, the critical energy release rate is also increased as the anisotropic parameter $\lambda$ increases. Since $\theta_{0}=0$, the slip plane is coincident

Fig. 3

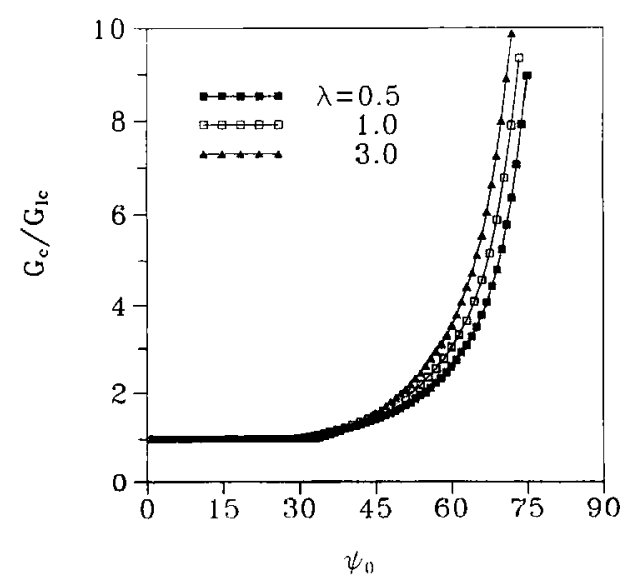

The non-dimensional critical energy release rate $G_{\mathfrak{c}} / G_{\mathrm{Ic}}$ against load phase angle $\psi_{0}$ for different values of $\lambda$ with $\theta_{0}=0, \rho_{K}=2.0$ and $\rho=0.99999$. 
Fig. 4

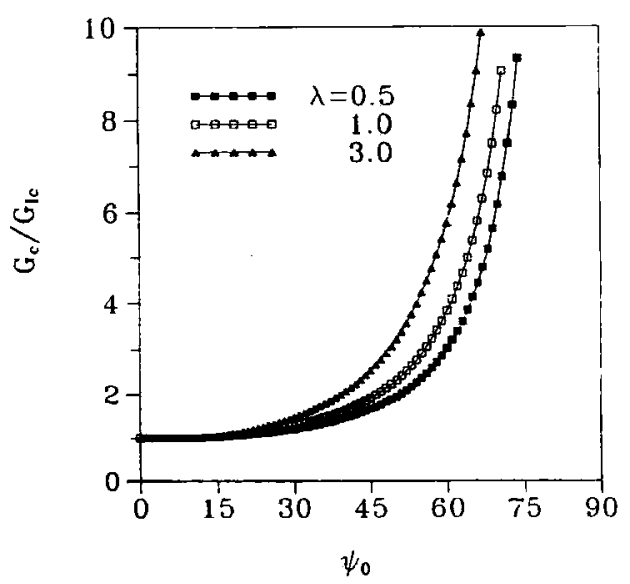

The non-dimensional critical energy release rate $G_{\mathrm{c}} / G_{\mathrm{Ic}}$ against load phase angle $\psi_{0}$ for different values of $\lambda$ with $\theta_{0}=0, \rho_{K}=5.0$ and $\rho=0.99999$.

with the crack plane. The crack extension is along $x_{1}$ direction. Suppose Young's modulus $E_{1}$ is fixed. The parameter $\lambda$ increase implies an increase in Young's modulus $E_{2}$, which results in an increase in the critical energy release rate.

The critical energy release rate against load phase angle $\psi_{0}$ is plotted in fig. 4 for different values of $\lambda$ for $\theta_{0}=0, \rho_{K}=5.0$ and $\rho=0.99999$. The elastic anisotropy effects on the energy release rates become pronounced. For example, at $\psi_{0}=60^{\circ}$, the elastic anisotropic value of $G_{\mathrm{c}} / G_{\mathrm{Ic}}$ is about 1.5 times that of the isotropic value.

If the slip plane is inclined at an angle $\theta_{0}=30^{\circ}$, the anisotropy effects on the energy release rates become tremendous. A typical example is shown in fig. 5 for

Fig. 5

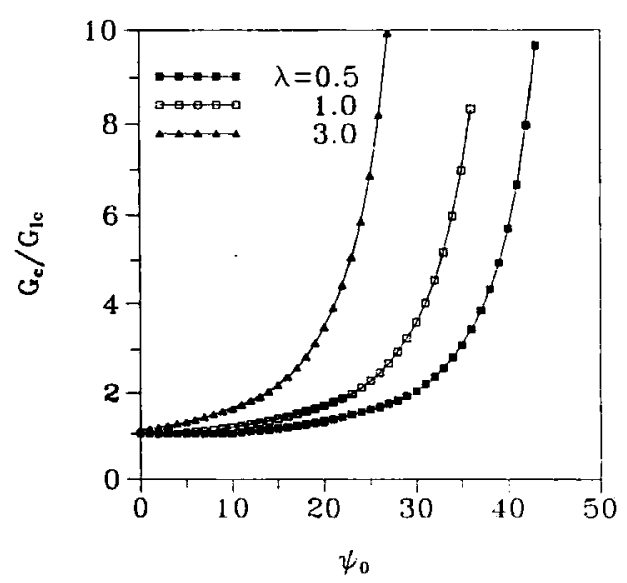

The relationship between the non-dimensional critical energy release rate $G_{\mathrm{c}} / G_{\mathrm{Ic}}$ and the load phase angle $\psi_{0}$ for different values of $\lambda$ with $\theta_{0}=30^{\circ} . \rho_{K}=3.0$ and $\rho=0.99999$. 
Fig. 6

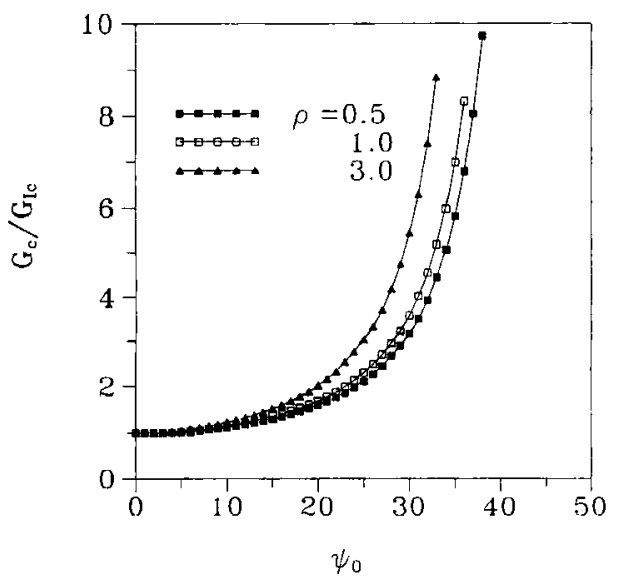

The effect of the anisotropic parameter $\rho$ on the critical energy release rate $G_{\mathrm{c}}$ for $\theta_{0}=30^{\circ}$, $\rho_{K}=3 \cdot 0$ and $\lambda=1 \cdot 0$.

$\rho_{K}=3.0$ and $\rho=0.99999$. In this case, the anisotropy parameter $\rho$ has pronounced effects also on the energy release rates as shown in fig. 6.

The general trends of the critical energy release rates and the total numbers of the emitted dislocations at the onset of cleavage are shown in figs. 7-12. The relation between the energy release rates and the load phase angle for different parameters $\rho_{K}$ is plotted in fig. 7 for $\theta_{0}=0, \lambda=3.0$ and $\rho=0.99999$. The total number $N$ of emitted dislocations at the onset of cleavage is shown in fig. 8 . These figures reveal that the critical energy release rates can increase to one to two magnitudes larger

Fig. 7

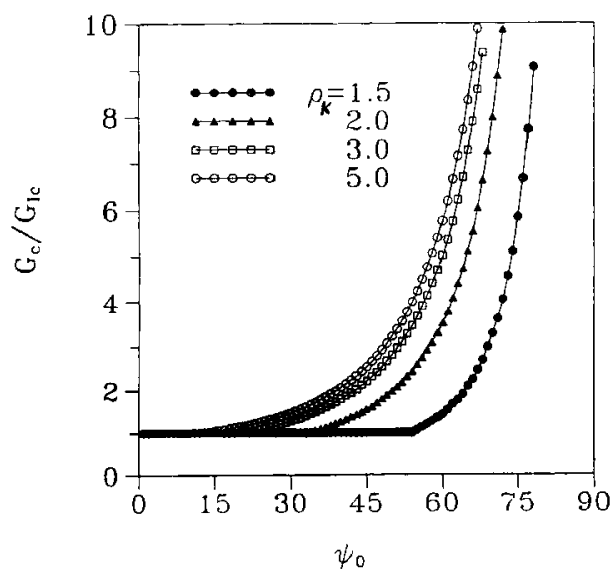

The effect of the parameter $\rho_{K}$ on the critical energy release rate $G_{\mathrm{c}}$ for $\theta_{0}=0, \rho=0.99999$ and $\lambda=3 \cdot 0$. 
Fig. 8

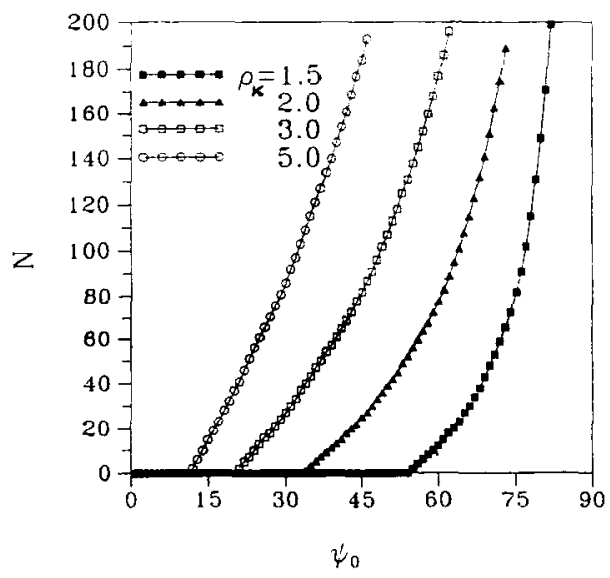

The effect of the parameter $\rho_{K}$ on the total number of emitted dislocations at onset of crack cleavage for $\theta_{0}=0, \rho=0.99999$ and $\lambda=3.0$.

Fig. 9

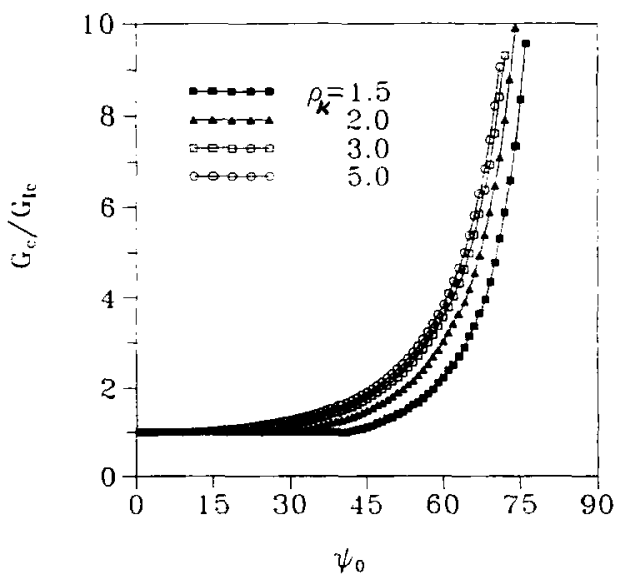

The effect of the parameter $\rho_{K}$ on the critical energy release rate $G_{\mathrm{c}}$ for $\theta_{0}=0, \rho=0.5$ and $\lambda=1 \cdot 0$.

than the $G_{\text {Ic }}$ due to the dislocation emissions. The total number $N$ can become several hundreds to several thousands when the load phase angle increases. The higher the total number $N$, the greater is the value of the critical energy release rate. One can see similar features in figs. 9 and 10 for $\lambda=1.0$ and $\rho_{\mathrm{s}}=0.5$.

\section{§4. RESULTS FOR FCC CRYSTAL}

In this section, we investigate the solutions for fcc crystal. Three specific orientations are considered here. For convenience, capital letters are used to label crack orientations. A crack on the $(010)$ plane with its tip along [ $[101]$ orientation is labelled 
Fig. 10

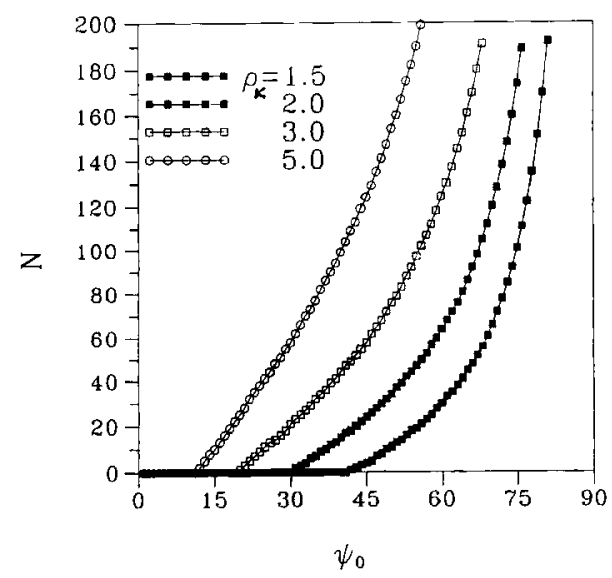

The effect of the parameter $\rho_{K}$ on the total number of emitted dislocations at onset of crack cleavage for $\theta_{0}=0, \rho=0.5$ and $\lambda=1.0$.

Fig. 11

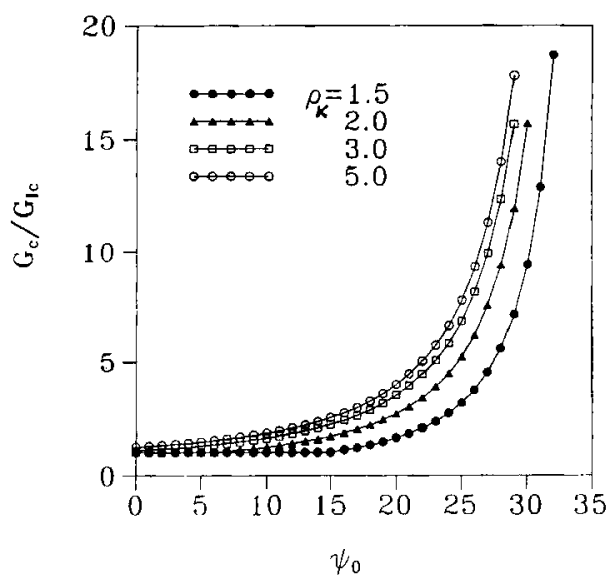

The non-dimensional critical energy release rate $G_{\mathrm{c}} / G_{\mathrm{Ic}}$ against load phase angle $\psi_{0}$ for different values of $\rho_{K}$ with $\theta_{0}=30^{\circ}, \lambda=3.0$ and $\rho=0.99999$.

as case A. A crack on the (111) plane with its tip along [101] is labelled case B. A crack on the plane $[\overline{1} 0 \overline{1}]$ ) with its tip along [101] is labelled case $C$.

From a micromechanism point view, dislocation emission from the crack tip in fcc materials consists of sequentially emitted partials rather than the full dislocations. For simplicity, we consider only the emission of paired partials which are formed in a definite order sequences and compose a full dislocation.

For fcc metal crystal, there are 12 possible slip systems, which consist of four $\{111\}$ slip planes with three $\langle 110\rangle$ slip directions on each plane. The crack and crystal orientation for case $\mathrm{A}$ are shown in fig. 13(a). The crack and the traces of the slip 
Fig. 12

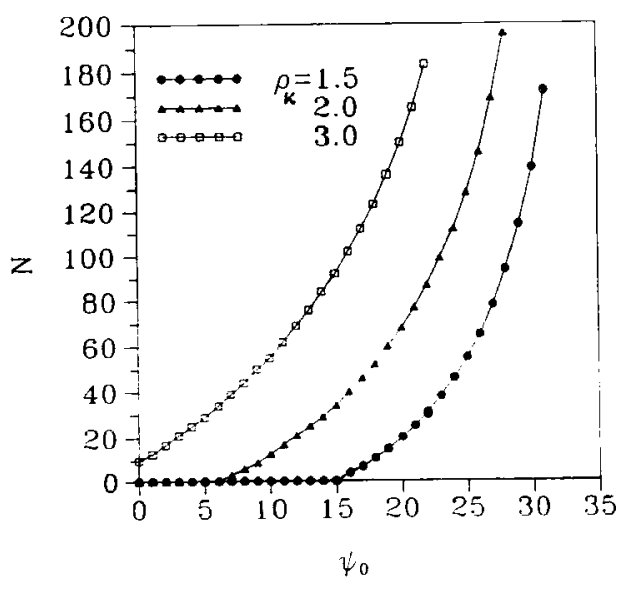

The total number $N$ of emitted dislocations at the onset of crack cleavage against load phase angle $\psi_{0}$ for different values of $\rho_{K}$ with $\theta_{0}=30^{\circ}, \lambda=3.0$ and $\rho=0.99999$.

Fig. 13

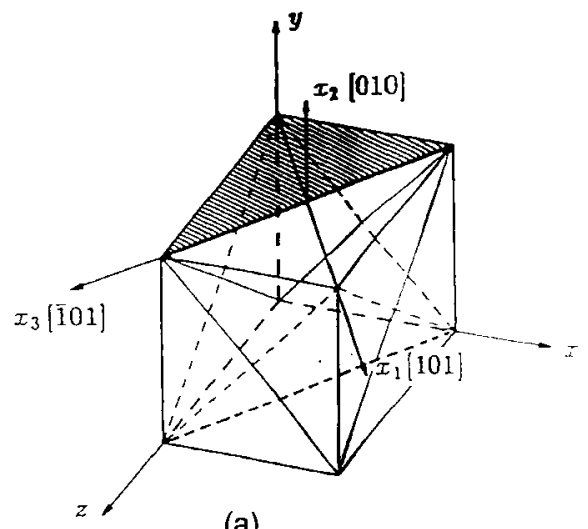

(a)

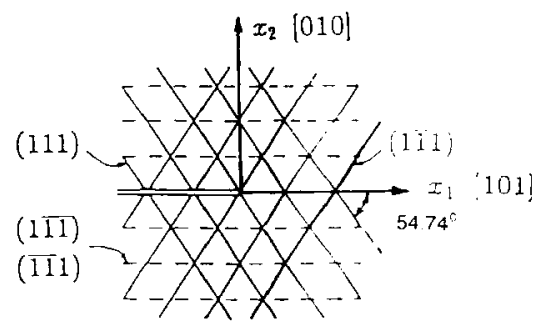

(b)

Fcc crystal. (a) Case A with a crack on (010) plane and its tip along [10i] $]$. (b) Plane-strain problem. The solid lines are the intersection of the slip planes (111) and (111) with the $x_{3}=0$ plane. The broken lines are the traces of simultaneous slip on the two planes

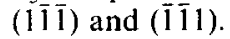


Fig. 14

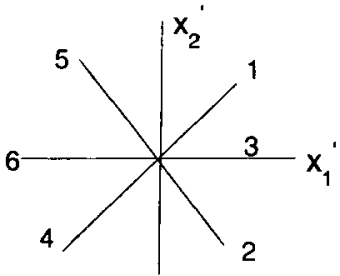

(a)

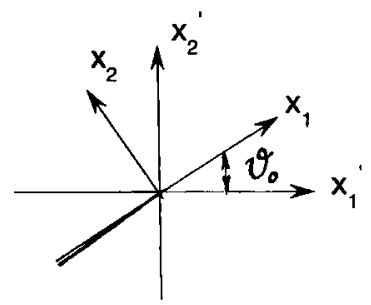

(b)

(a) Effective slip systems. (b) Rotation from the coordinate system o $x_{1}^{\prime} x_{2}^{\prime}$ to the coordinate system $o x_{1} x_{2}$.

planes in the $x_{1}-x_{2}$ plane are depicted in fig. 13(b). The solid lines are the intersections of the slip planes (111) and (111) with the $x_{3}=0$ plane. Simultaneously slip on the two slip systems $[1 \overline{1} 0](111)$ and $[0 \overline{1} 1](111)$ will cause an effective slip on the direction which is parallel to the intersection of the slip plane (111) with the $x_{3}=0$ plane. Similarly we have a second effective slip direction, as shown in fig. 13(b). Since the plane $(010)$ is a cleavage plane and not a slip plane, the $(010)[101]$ is not a permitted slip system for the plane-strain problem. Now we only have two effective slip systems for in-plane deformation. If a slip with opposite direction is considered as another slip system, then we have four slip systems altogether. For convenience, each slip system is labelled with a number $M(=1,2,4,5)$ as shown in fig. 14. The crack propagations in direction [101] and direction [101] on the cleavage plane (101) are labelled $M=3$ and $M=6$ respectively. Of course each slip system is also considered as a possible crack propagation system. Then we have six crack propagation directions altogether. For a fec crystal, the crystal principal axes are in the $x, y$ and $z$ directions. In these principal axes, the fcc crystal is a transverse isotropic material. From the coordinate system oxyz to the coordinate system o $x_{1} x_{2} x_{3}$ there is an in-plane coordinate rotation:

where $\phi=45^{\circ}$.

$$
\mathbf{R}=\left[\begin{array}{ccc}
\cos \phi & 0 & \sin \phi \\
0 & 1 & 0 \\
-\sin \phi & 0 & \cos \phi
\end{array}\right]
$$

After some manipulations, one can verify that the stress vector and the strain vector transform as

$$
\boldsymbol{\sigma}=\mathbf{Q} \boldsymbol{\sigma}_{\mathrm{c}}, \quad \varepsilon=\mathbf{Q} \varepsilon_{\mathrm{c}}
$$

where $\sigma$ and $\varepsilon$ are the stress and strain vectors respectively in the coordinate system o $x_{1} x_{2} x_{3}$, and $\sigma_{\mathrm{c}}$ and $\varepsilon_{\mathrm{c}}$ are the stress and strain vectors respectively in the principal coordinate system oxyz:

$$
\begin{array}{rlrl}
\boldsymbol{\sigma} & =\left[\sigma_{11}, \sigma_{22}, \sigma_{33}, \sigma_{23}, \sigma_{31}, \sigma_{12}\right]^{\mathrm{T}}, & \boldsymbol{\varepsilon} & =\left[\varepsilon_{11} \varepsilon_{22}, \varepsilon_{33}, \gamma_{23}, \gamma_{31}, \gamma_{12}\right]^{\mathrm{T}}, \\
\boldsymbol{\sigma}_{\mathrm{c}} & =\left[\sigma_{x x}, \sigma_{y y}, \sigma_{z z}, \sigma_{z x}, \sigma_{x y}\right]^{\mathrm{T}}, & \boldsymbol{\varepsilon}_{\mathrm{c}}=\left[\varepsilon_{x x}, \varepsilon_{y y}, \varepsilon_{z z}, \gamma_{y z}, \gamma_{z x}, \gamma_{x y}\right]^{\mathrm{T}},
\end{array}
$$




$$
\mathbf{Q}=\left[\begin{array}{cccccc}
\cos ^{2} \phi & 0 & \sin ^{2} \phi & 0 & \cos \phi \sin \phi & 0 \\
0 & 1 & 0 & 0 & 0 & 0 \\
\sin ^{2} \phi & 0 & \cos ^{2} \phi & 0 & -\cos \phi \sin \phi & 0 \\
0 & 0 & 0 & \cos \phi & 0 & -\sin \phi \\
-\sin (2 \phi) & 0 & \sin (2 \phi) & 0 & \cos (2 \phi) & 0 \\
0 & 0 & 0 & \sin \phi & 0 & \cos \phi
\end{array}\right]
$$

According to the constitutive law, we have

$$
\boldsymbol{\sigma}_{\mathrm{c}}=\mathbf{S} \boldsymbol{\varepsilon}_{\mathrm{c}}
$$

From the above equations, one obtains

$$
\boldsymbol{\sigma}=\mathbf{Q S Q}^{\mathrm{T}} \boldsymbol{\sigma} \quad \mathbf{s}=\mathbf{Q S Q}^{\mathbf{T}},
$$

where $\mathbf{s}$ and $\mathbf{S}$ are the elastic compliance tensors of the fcc material in the coordinate system $0 x_{1} x_{2} x_{3}$ and principal coordinate system $o x y z$ respectively. For a fcc crystal, the compliance tensor $\mathbf{S}$ is

$$
\mathbf{S}=\left[\begin{array}{cccccc}
S_{11} & S_{12} & S_{12} & 0 & 0 & 0 \\
S_{12} & S_{11} & S_{12} & 0 & 0 & 0 \\
S_{12} & S_{12} & S_{11} & 0 & 0 & 0 \\
0 & 0 & 0 & S_{44} & 0 & 0 \\
0 & 0 & 0 & 0 & S_{44} & 0 \\
0 & 0 & 0 & 0 & 0 & S_{44}
\end{array}\right]
$$

Substituting eqn. (44) into eqn. (46) and carrying out some manipulation, we obtain

$$
\begin{aligned}
& s_{11}=S_{11}\left[1+2(\rho-1) \cos ^{2} \phi \sin ^{2} \phi\right], \\
& s_{12}=S_{12}, \\
& s_{13}=S_{12}-2(\rho-1) \cos ^{2} \phi \sin ^{2} \phi S_{11}, \\
& s_{14}=s_{16}=0, \\
& s_{15}=S_{11}(\rho-1) \cos (2 \phi) \sin (2 \phi), \\
& s_{22}=S_{22}=S_{11}, \\
& s_{23}=S_{12}, \\
& s_{24}=s_{25}=s_{26}=0, \\
& s_{33}=s_{11}, s_{34}=s_{36}=0, \\
& s_{35}=\frac{1}{2}(\rho-1) \sin (4 \phi), \\
& s_{44}=S_{44}, s_{45}=s_{46}=0 \\
& s_{55}=S_{44}-S_{11} 2(\rho-1) \sin ^{2}(2 \phi), \quad s_{56}=0, \\
& s_{66}=S_{44} .
\end{aligned}
$$


Substituting $\phi=45^{\circ}$ into the above equation, we find that

$$
\begin{aligned}
& s_{14}=s_{15}=s_{16}=0, \quad s_{24}=s_{25}=s_{26}=0, \\
& s_{34}=s_{35}=s_{36}=0, \quad s_{45}=s_{46}=s_{54}=0, \\
& s_{56}=0 .
\end{aligned}
$$

Hence in the coordinate system o $x_{1} x_{2} x_{3}$ the fcc crystal is an orthotropic solid. The characteristic equation for the characteristic numbers is

The solution is

$$
\frac{1}{2}(1+\rho) \mu^{4}+2 \rho \mu^{2}+\frac{1}{\lambda}=0 .
$$

$$
\begin{aligned}
\mu_{1} & =\mathrm{i} \lambda^{-1 / 4}\left(n^{*}+m^{*}\right), & \mu_{2}=\mathrm{i} \lambda^{-1 / 4}\left(n^{*}-m^{*}\right), & 1 \leqslant \rho \leqslant \infty, \\
\mu & =\lambda^{-1 / 4}\left(\mathrm{i} n^{*}+m^{*}\right), & \mu_{2}=\lambda^{-1 / 4}\left(\mathrm{i} n^{*}-m^{*}\right), & -1 \leqslant \rho \leqslant 1, \\
n^{*} & =\left[\frac{1}{2}\left(1+\frac{\rho}{n}\right)\right]^{1 / 2}, & m^{*}=\left[\frac{1}{2}\left(1-\frac{\rho}{n}\right)\right]^{1 / 2} . &
\end{aligned}
$$

\subsection{Calculation procedure}

The calculation is carried out step by step. Each step has only one dislocation emission from the crack tip. At each step, we know the stress intensity factors $k_{1}$ and $k_{2}$, the local stress intensity factors $k_{1}^{\text {tip }}$ and $k_{2}^{\text {tip }}$ and the local stress intensity factors $K_{1}^{\text {tip }}(M)$ and $K_{2}^{\text {tip }}(M)$, where $M$ indicates the corresponding slip system in which the stress intensity factors $K_{1}(M)$ and $K_{2}(M)$ are defined. Now we search the load increment at which a dislocation will fully nucleate on the most stressed slip system.

The stress intensity factors $k_{1}$ and $k_{2}$ are referred to as the external loads. The load phase angle $\psi_{0}$ is defined as

$$
\tan \psi_{0}=k_{1} / k_{2}
$$

which also describes the mode mixity.

For a given load phase angle $\psi_{0}$, we have

$$
k_{1}=\tan \psi_{0} k_{2}, \quad \Delta k_{1}=\tan \psi_{0} \Delta k_{2}
$$

Suppose that the external loads increase from $k_{1}$ and $k_{2}$ to $k_{1}+\Delta k_{1}$ and $k_{2}+\Delta k_{2}$. For slip system $M$, the increment $\Delta K_{1}^{\text {tip }}(M)$ of the local stress intensity factor $K_{1}^{\mathrm{tip}}(M)$ is given by

$$
\Delta K_{1}^{\mathrm{tip}}(M)=f_{11} \Delta k_{1}+f_{12} \Delta k_{2}=\left(f_{11} \tan \psi_{0}+f_{12}\right) \Delta k_{2},
$$

where the coefficients $f_{i j}$ depend on the slip system. The increment $\Delta k_{2}(M)$ at which a dislocation will fully nucleate at the slip system $M$ can be determined as follows:

$$
\Delta k_{2}(M)=\frac{K_{\mathrm{IIe}}(M)-K_{1}^{\mathrm{tip}}(M)}{f_{11} \tan \psi_{0}+f_{12}} .
$$

Obviously the real load increment $\left(\Delta k_{2}\right)_{0}$ is given by the lowest value $\min \left[\Delta k_{2}(M)\right]$, which corresponds to the most highly shear stressed slip system. During this loading process, crack cleavage may occur. Hence we need to check carefully whether or not the cleavage according to eqn. (24) will occur at a certain stage. We divide this 
increment step into 100 substeps. At each substep, we calculate the increments $\Delta K_{1}^{\mathrm{tip}}(M)$ and $\Delta K_{2}^{\mathrm{tip}}(M)$ for each slip system:

$$
\begin{aligned}
& \Delta K_{1}^{\text {tip }}(M)=f_{11} \Delta k_{1}+f_{12} \Delta k_{2}, \\
& \Delta K_{2}^{\text {tip }}(M)=f_{21} \Delta k_{1}+f_{22} \Delta k_{2} .
\end{aligned}
$$

Then the local stress intensity factors of the slip system $M$ can be expressed as

$$
K_{1}^{\mathrm{tip}}(M)+\Delta K_{1}^{\mathrm{tip}}(M) \text { and } K_{2}^{\mathrm{tip}}(M)+\Delta K_{2}^{\mathrm{tip}}(M) .
$$

Substituting the above values of the local stress intensity factors into eqn. (24), we check whether or not this equation is met. If eqn. (24) is met for one slip system at a certain stage we make sure that $K_{2}^{\mathrm{tip}}(M)$ is positive; then cleavage should occur on this slip system before a new dislocation emission. Then the calculation is finished. (In order to obtain a more accurate value of the critical load, one can divide the substeps, which are around the critical load, into 100 subsubsteps and then calculate again.) If in the whole increment loading process, we cannot find any possibility of meeting eqn. (24), a new dislocation will be emitted from the crack tip along the most shear stressed slip system $M_{0}$. After this dislocation emission, the local stress intensity factors are shielded by this emitted dislocation. Hence we obtain

$$
\begin{aligned}
& \Delta k_{1}^{\text {tip }}=\tan \psi_{0}\left(\Delta k_{2}\right)_{0}-c_{1}\left(M_{0}\right) K_{\text {Ile }}, \\
& \Delta k_{2}^{\text {tip }}=\left(\Delta k_{2}\right)_{0}-c_{2}\left(M_{0}\right) K_{\text {IIe }},
\end{aligned}
$$

where the first term of the right-hand side of the above equation is the contribution due to the external loading, and the second term is the shielding contribution by the emitted dislocation. Then we can start the next load increment. As the sequence is repeated, the whole calculation is carried out.

\subsection{Calculation results}

All the results given here are for a fcc aluminium crystal. The material parameters are $\lambda=1.0$ and $\rho=0.72$ for the plane-strain case (Suo 1989).

\subsubsection{Case $A$}

A typical calculation results for a fcc aluminium crystal are shown in figs. 15 and 16. The other materials parameters are chosen as

$$
\begin{aligned}
K_{\mathrm{Ic}}(M) & =K_{\mathrm{Ic}}, \quad M=1,2, \ldots, 6, \\
K_{\mathrm{IIe}}(M) & =K_{\mathrm{IIe}}, \quad M=1,2,4,5, \\
\rho_{K} & =2 \cdot 5, \quad q_{0}=\frac{b}{\left(S_{11} S_{22}\right)^{1 / 2}\left(2 \pi r_{\mathrm{c}}\right)^{1 / 2} K_{\mathrm{IIe}}}=0.054 .
\end{aligned}
$$

The energy release rate $G_{\mathrm{c}} / G_{\mathrm{Ic}}$ against load phase angle $\psi_{0}$ is plotted in fig. 15 . When $\psi_{0}<11.4^{\circ}$ there is no dislocation emission. The crack cleavage propagation occurs along the crack plane without any dislocation emission. When $11.4^{\circ}<\psi_{0}<17.4^{\circ}$ there is a number of dislocation emissions along the first slip system before crack cleavage extension. The critical energy release rate $G_{\mathrm{c}}$ and the total number $N$ of emitted dislocations increase rapidly when $15.0^{\circ}<\psi_{0}<17.4^{\circ}$.

When $17.5^{\circ}<\psi_{0}<20.5^{\circ}$, crack branching occurs along the second slip plane after a number of dislocation emissions along the first slip plane. The critical energy 
Fig. 15

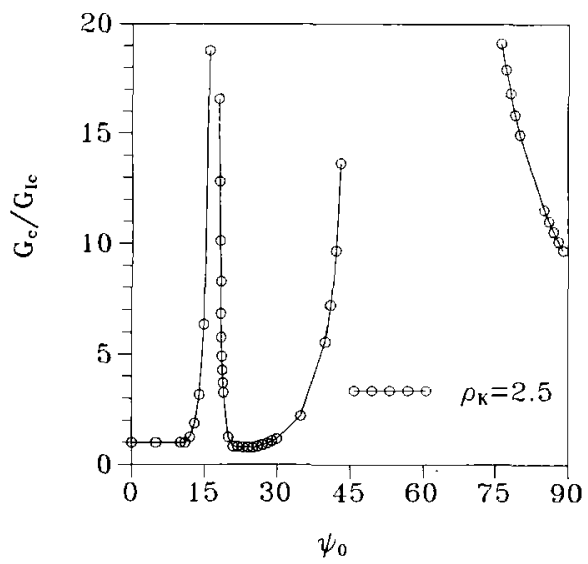

The non-dimensional critical energy release rate $G_{\mathrm{c}} / G_{\mathrm{Ic}}$ against load phase angle $\psi_{0}$ for fcc single-crystal aluminium (case A) with $\rho_{K}=2 \cdot 5$.

Fig. 16

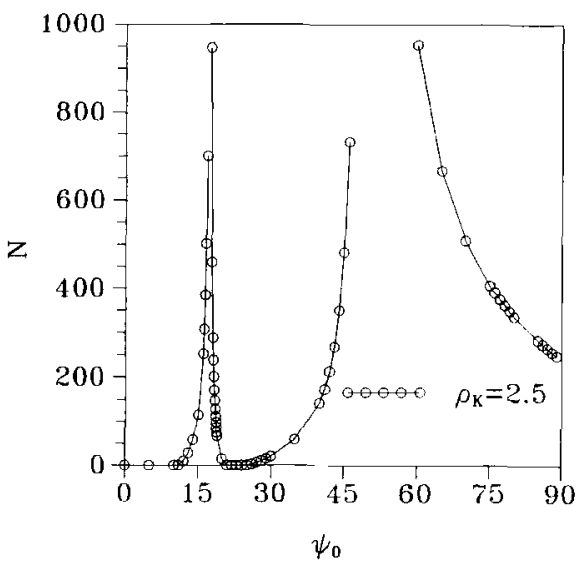

The total number $N$ of emitted dislocations at the onset of the crack cleavage against load phase angle $\psi_{0}$ for fcc single-crystal aluminium (case A) with $\rho_{K}=2 \cdot 5$.

release rate $G_{\mathrm{c}}$ decreases rapidly with increasing $\psi_{0}$ in this region. The aluminium crystal behaves as a perfect brittle material when $20.6^{\circ}<\psi_{0}<25.4^{\circ}$. When $25.5^{\circ}<\psi_{0}<48.0^{\circ}$, the fourth slip system is active. The crack branches into the second slip plane after a number of dislocation emissions along the fourth slip plane. The energy release rate $G_{\mathrm{c}}$ reaches the second peak at $\psi_{0} \approx 49^{\circ}$. The crack will still branch into the second slip plane in the region $49^{\circ}<\psi_{0}<90^{\circ}$, but the critical energy release rate $G_{\mathrm{c}}$ will decrease with increasing $\psi_{0}$.

The total number $N$ of emitted dislocations before crack propagation is depicted in fig. 16. The tendency is consistent with fig. 15. The higher the total number $N$, the higher is the critical energy release rate $G_{\mathrm{c}}$. 
Fig. 17

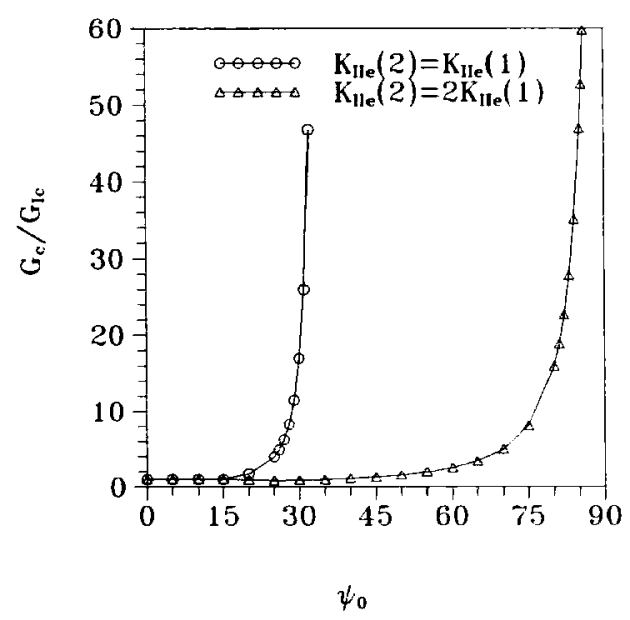

The non-dimensional critical energy release rate $G_{\mathrm{c}} / G_{\mathrm{Ic}}$ against load phase angle $\psi_{0}$ for fcc single-crystal aluminium (case B) with $\rho_{K}=2 \cdot 5$.

\subsubsection{Case $B$}

There is an additional in-plane rotation from the coordinate system $o x_{1}^{\prime} x_{2}^{\prime} x_{3}^{\prime}$ to the coordinate system $o x_{1} x_{2} x_{3}$ as shown in fig. $14(b)$. The rotation angle $\vartheta_{0}=$ $54.74^{\circ}$. The crack plane is parallel to the first slip plane. When $0^{\circ}<\psi_{0}<15 \cdot 0^{\circ}$, there is crack brittle propagation along the first slip plane without any dislocation emission as shown in figs. 17 and 18 .

When $15.0^{\circ}<\psi_{0}<30.0^{\circ}$, crack extension occurs in the first slip plane after a number of dislocation emissions along the second slip plane. When $31 \cdot 0^{\circ}<$ $\psi_{0}<344^{\circ}$, the first slip plane is active. The crack extends along the first slip

Fig. 18

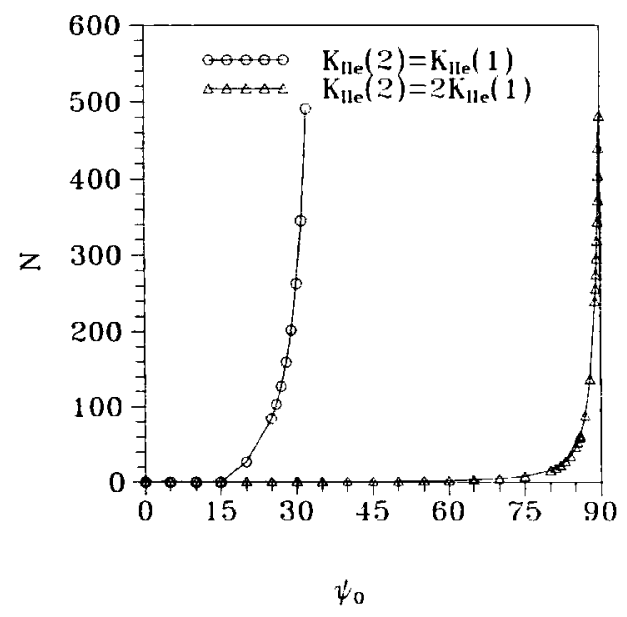

The total number $N$ of emitted dislocations at the onset of crack cleavage against load phase angle $\psi_{0}$ for fcc single-crystal aluminium (case B) with $\rho_{K}=2.5$. 
plane after a number of dislocation emissions along the first slip plane and the second slip plane. Where $34^{\circ}<\psi_{0}$, the energy release rate and the total number $N$ of emitted dislocations increase rapidly. For example, for $\psi_{0}=34 \cdot 4^{\circ}, G_{\mathrm{c}} / G_{\mathrm{Ic}}=7107$ and $N=6941$.

As pointed by Zhou et al. (1994) and Xu et al. (1995), the ledge generation has a significant effect on dislocation nucleation and emission. In order to evaluate the ledge generation effect, we can give up the assumption that all $K_{\text {IIe }}(M)=K_{\text {IIe }}$, $M=1,2,4,5$. For example, we can assume that

$$
\begin{aligned}
K_{\mathrm{Ic}}(M) & =K_{\mathrm{Ic}}, & & M=1,2, \ldots, 6 . \\
K_{\mathrm{IIe}}(M) & =K_{\mathrm{IIe}}, & & M=1,4 . \\
K_{\mathrm{IIe}}(M) & =2 K_{\text {IIe }}, & & M=2,5 .
\end{aligned}
$$

The corresponding calculation results are also shown in figs. 17 and 18 (solid curve with open triangles). When $0^{\circ}<\psi_{0}<15 \cdot 0^{\circ}$, the results are the same as the results with the assumption that all $K_{\mathrm{IIe}}(M)=K_{\mathrm{II}}, M=1,2,4,5$. However, when $15.0^{\circ}<\psi_{0}$ the results with the assumption (57) are completely different from the results with the assumption (56). The materials become much more brittle since the second and the fifth slip systems have a high resistance to dislocation emission along these slip planes. One cannot find any dislocation emission along these slip planes before crack extension along the cleavage plane $(010)$ in the direction [101] when $17.0^{\circ}<\psi_{0}<90 \cdot 0^{\circ}$. Crack branching occurs into the cleavage plane $(010)$ in the direction [101] after a number of dislocation emissions along the first slip plane when $27 \cdot 0^{\circ}<\psi_{0}<90 \cdot 0^{\circ}$.

\subsubsection{Case $C$}

There is an additional in-plane rotation from the coordinate system $o x_{1}^{\prime} x_{2}^{\prime} x_{3}^{\prime}$ to the coordinate system $o x_{1} x_{2} x_{3}$ as shown in fig. $14(b)$. The rotation angle $\vartheta_{0}=90^{\circ}$. The energy release rate $G_{\mathrm{c}} / G_{\mathrm{Ic}}$ against load phase angle $\psi_{0}$ is plotted in fig. 19 . The

Fig. 19

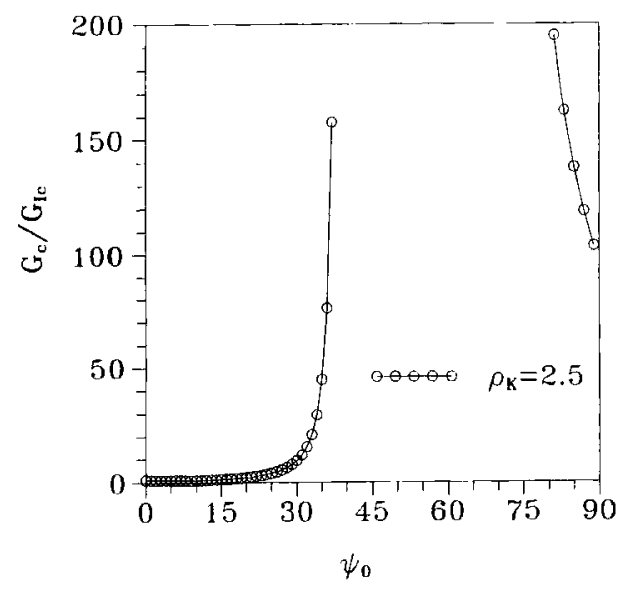

The non-dimensional critical energy release rate $G_{\mathrm{c}} / G_{\mathrm{Ic}}$ against load phase angle $\psi_{0}$ for fcc single-crystal aluminium (case C) with $\rho_{K}=2 \cdot 5$. 
Fig. 20

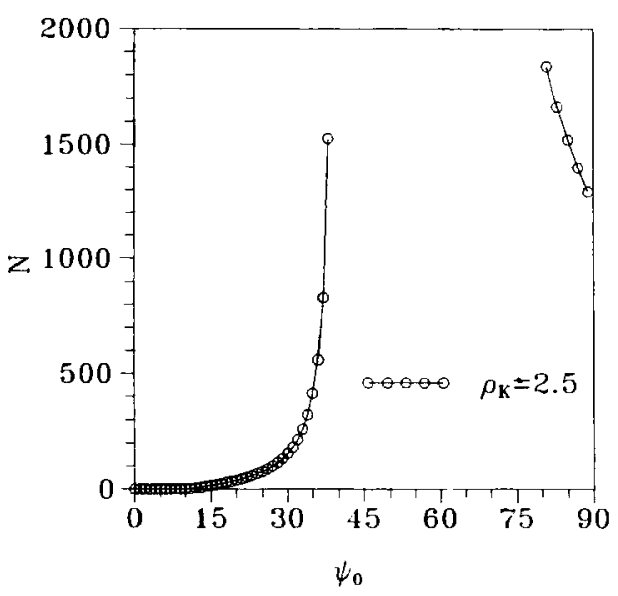

The total number $N$ of emitted dislocations at the onset of crack cleavage against load phase angle $\psi_{0}$ for the fcc single-crystal aluminium (case C) with $\rho_{K}=2 \cdot 5$.

total number $N$ of emitted dislocations before crack extension is shown in fig. 20 . The crack branches into the first slip plane without any dislocation emission when $0^{\circ}<\psi_{0}<9.8^{\circ}$. When $9.8^{\circ}<\psi_{0}<39.0^{\circ}$ there are a number of dislocation emissions along the fifth slip plane before crack branching into the first slip plane. The critical energy release rate increases rapidly when $35^{\circ}<\psi_{0}$. When $67^{\circ}<\psi_{0}<90^{\circ}$ the critical energy release rate decreases rapidly with increasing $\psi_{0}$. The crack branches into the cleavage plane (010) in the [101] direction after numerous dislocation emissions along the second slip plane and the fifth slip plane. For example, when $\psi_{0}=75.0^{\circ}$, crack branching occurs along direction [101] on the cleavage plane (010) after 2554 dislocation emissions along the second slip plane and 132 dislocation emissions along the fifth slip plane.

\section{§5. Conclusions AND Discussion}

From this study we can draw several conclusions.

(1) A dislocation theory of fracture criterion for a mixed dislocation emission and cleavage process in an anisotropic solid is developed in this paper. The explicit formula for the critical condition of crack cleavage in an anisotropic solid after a number of dislocation emissions is established. The effects of elastic anisotropy, crack geometry and load phase angle on the critical energy release rates and the total number of the emitted dislocations at the onset of cleavage are analysed.

(2) The critical energy release rates can increase to one or two magnitudes larger than the surface energy owing to the dislocation emission. The ledge generation has significant effects not only on the dislocation nucleation and emission along an inclined slip plane but also on the competition process between dislocation emission and crack cleavage. A typical analysis of ledge generation effects for case $\mathbf{B}$ has clearly shown that the materials become 
much more brittle since the dislocation emission along inclined slip planes has a higher resistance.

(3) The elastic anisotropy and the crystal orientation have significant effects on the critical energy release rates. The anisotropic values can be several times larger than the isotropic value in one crack orientation. The values may be as much as $40 \%$ less than the isotropic value in another crack orientation and with another anisotropy parameter.

(4) The present theory is successfully applied to a fcc single crystal. An edge dislocation can be emitted from the crack tip along the most shear stressed slip plane. Crack cleavage can occur along the slip plane, in which the energy release rate reaches the maximum value, after a number of dislocation emissions. The calculation clearly shows that the crack orientation and the load phase angle have significant effects on the crystal brittle-ductile behaviour.

Many simplified assumptions have been introduced in the present analyses. The dislocation emission actually takes place in the three-dimensional geometry by a dislocation loop. The present analyses are only concerned the two-dimensional description. The effects of thermal activity of the molecules on the dislocation emission and the nonlinear interaction between the shear stress and the normal stress in the cohesive zone are neglected here. In order to obtain a better understanding of the brittle-ductile behaviour of the crystal, further research is needed.

\section{ACKNOWLEDGEMENT}

This work was supported by The Natural Sciences Foundation of China.

Atkinson, C., 1966, Int. J. Fract., 2, 567.

\section{REFERENCES}

Asaro, R. J., 1975, J. Phys. F, 5, 2249.

BeLtZ, G. E., and RICE, J. R., 1991, Modeling the Deformation of Crystalline Solids, edited by C. L. Terry, D. R. Anthony, P. S. Follansbee and G. S. Daehn, p. 457.

LekhnitskiI, S. G., 1963, Theory of Elasticity of an Anisotropic Body (San Francisco, California: Holden-Day).

Eshelby, J. D., Read, W. T., and Shockley, W., 1953, Acta metall., 1, 251.

Lin, I. H., and Thomson, R. M., 1986, Acta metall., 34, 187.

OHR, S. M., 1985, Mater. Sci. Engng., 72, 1.

Rice, J. R., 1992, J. Mech. Phys. Solids, 40, 239.

Rice, J. R., Beltz, G. E., and Sun, Y., 1992, Topics in Fracture and Fatigue, edited by A. S. Argon (Berlin: Springer), p. 1.

Rice, J. R., and Thomson, R. M., 1974, Phil. Mag., 29, 73.

SCHOECK, G., 1991, Phil. Mag., 63, 111.

Sinclair, J. E., and Finnis, M. W., 1983, Atomics of Fracture, edited by R. M. Latanision and J. R. Pickens, p. 1047.

Stroh, A. N., 1958, Phil. Mag., 7, 625.

Sir, G. C., Paris, P. C., and Irwin, G. R., 1965, Int. J. Fract. Mech., 1, 189.

Sun, Y., and Beltz, G. E., 1994, J. Mech. Phys. Solids, 42, 1905.

Suo, Z., 1989, PhD Thesis, Harvard University, Cambridge, Massachusetts; 1990, Proc. $R$. Soc. A, 427, 331.

WANG, T. C., 1994, Int. J. Solids Struct., 31, 629; 1995, Int. J. Fract., 69, 295; 1996, Phil. Mag. A, 74, 983 .

Wang, T. C., Shin, C. F., and Suo, Z., 1992, Int. J. Solids Struct., 29, 327.

Xu, G., Argon, A. S., and Ortiz, M., 1995, Phil. Mag., A, 72, 415.

Zhou, S. J., Carlsson, A. E., and Thomson, R., 1994, Phys. Rev. Lett., 72, 852. 